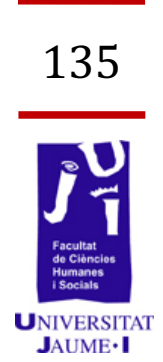

\title{
La Arquitectura Romana en la Península Ibérica Los Edificios de Poder
}


En el presente trabajo de investigación analizaremos la arquitectura de poder propia del mundo romano; pero antes de abordar directamente este tema, trataremos de definir la arquitectura de poder desde una visión más general, que nos permita analizar su evolución en las distintas épocas históricas.

En segundo lugar, nos acercaremos tanto a Roma, la Città Eterna, como a otros lugares dominados en su momento por los romanos, para centrarnos finalmente en aquellos monumentos $\mathrm{y} / \mathrm{o}$ enclaves arqueológicos de gran importancia, analizarlos e identificar el tipo de edificio para posteriormente detallar la influencia de éste sobre otros que podamos encontrar en la Península ibérica.

Finalmente, en el último apartado analizaremos alguno de los ejemplos de los más importantes edificios de poder construidos en Hispania en época romana, a saber entre foros, templos, teatros, termas, etc.

Palabras Clave: Arquitectura, poder, Roma, Hispania, foros, templos, teatros, termas.

\section{Introducción y objetivos}

El objetivo fundamental de este estudio es realizar un análisis general sobre la evolución de los distintos edificios de poder propios de la cultura romana, para proponer finalmente un modelo de propuesta de catalogación. Tras observar los modelos constructivos más influyentes en Roma, nos centraremos en analizar algunos de los restos arquitectónicos más interesantes de época romana localizados en la Península Ibérica, que podemos clasificar como edificios de poder, por ejemplo los foros, templos, termas, villas, teatros, circos y anfiteatros.

Antes de abordar el corpus principal de nuestro estudio, vamos a tratar lo que es la arquitectura. Según la RAE, el término arquitectura hace referencia al "arte de proyectar y construir edificios"; por otra parte, la palabra poder, dentro de todas las acepciones o significados que tiene, el que más nos interesa es el de "dominio, imperio, facultad y jurisdicción que alguien tiene para mandar o ejecutar algo". Por lo tanto la composición o unión de ambas palabras, la podemos resumir o definir como aquel arte que es proyectado, construido o mandado construir por un particular o un conjunto de personas sobre las que recae el poder.

A lo largo de la historia muchos han tratado de definir lo que es la arquitectura, de ahí que debamos recurrir a los libros de arquitectura de Vitrubio, como fundamento de nuestra investigación, pues los más antiguos que se conocen y fueron escritos en el siglo I a.C. y descubiertos a inicios del siglo XV. Este fue el principal referente para conocer la arquitectura y el urbanismo clásico. 
Hoy en día, existen distintas formas de catalogar y aportar información acerca de los edificios romanos de época antigua, como por ejemplo: a través de sus restos arqueológicos, donde usualmente hallamos las cimentaciones y restos de la cultura material, que nos ayuda a identificar su funcionalidad; otra forma sería a través de las monedas halladas en las excavaciones arqueológicas [Figura 1], ya que en su anverso/reverso aparece el benefactor y suelen representarse edificios muy representativos de las sociedad romana. Además, no podemos olvidarnos, de las pinturas del siglo XVIII y XIX [Figura 2], donde pintores como Pannini, Codazzi o Canaletto acudían a Roma para admirar sus ruinas, para posteriormente plasmarlas en sus obras.

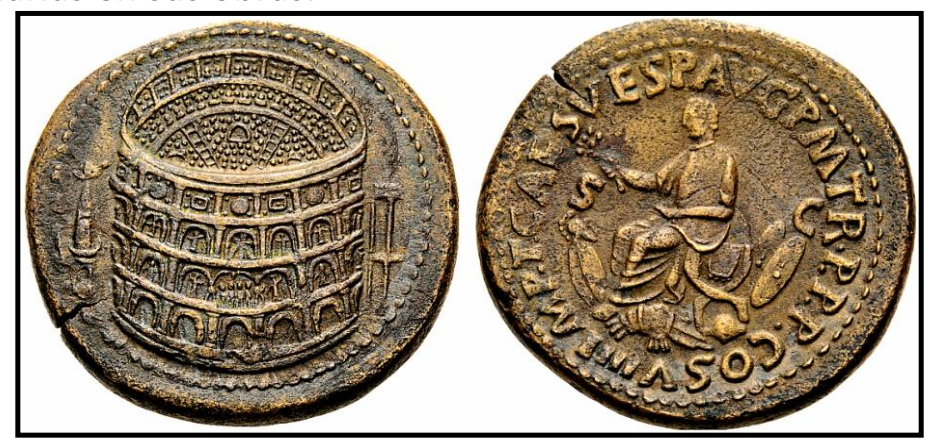

Figura 1: Sestercio acuñado sobre el año 80 d.C. por el emperador Tito, con el Coliseo en su anverso. Fuente: https://lh5.googleusercontent.com

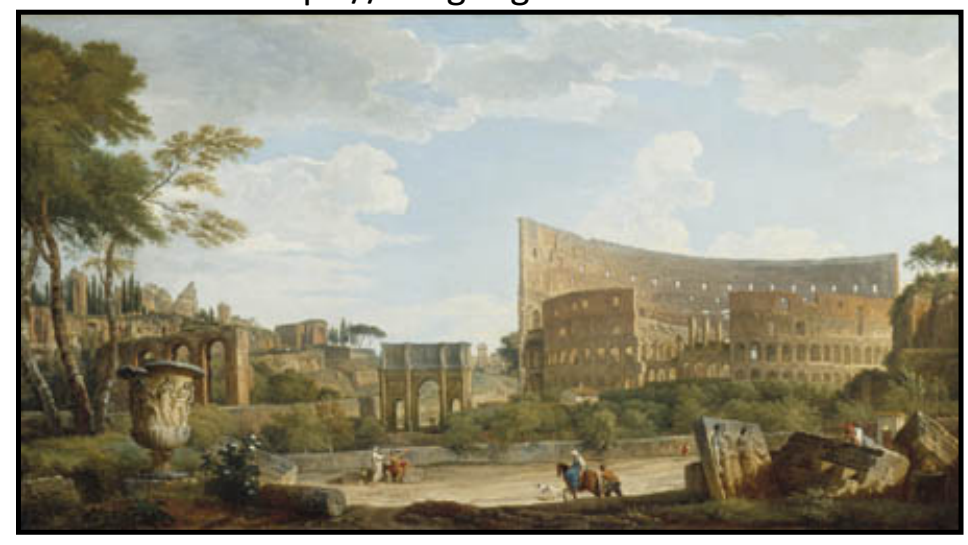

Figura 2: Vista de las ruinas del Coliseo, el arco de Constantino y otros restos del Foro, obra de Giovanni Pannini (1735). Fuente: http://www.foroxerbar.com.

Así pues, todas estas formas de reconocer, catalogar y aportar información acerca de los edificios romanos de época antigua nos arrojan luz de cómo perduraron en el tiempo, pero nuestro siguiente paso consistirá en saber cómo nace esta arquitectura y lo que hizo que esta cultura arquitectónica se expandiera por el vasto territorio dominado por los romanos.

Tal y como describe Marín Sánchez, la arquitectura romana tuvo como punto de partida el pasado helenístico y etrusco, alcanzando un carácter propio hacia el final del primer milenio a. C. (Marín Sánchez, 2000: 123); es decir, el autor quiere reflejar que era heredera de la cultura etrusca cómo 
podemos observar en el templo más antiguo conservado, que es el de Veio (Italia) [Figura 3 y 4], el cual data aproximadamente sobre el siglo VI-V A. C.; por lo tanto, podemos afirmar que la cultura romana fue la sucesora tanto de la cultura como del mundo helénico y etrusco.
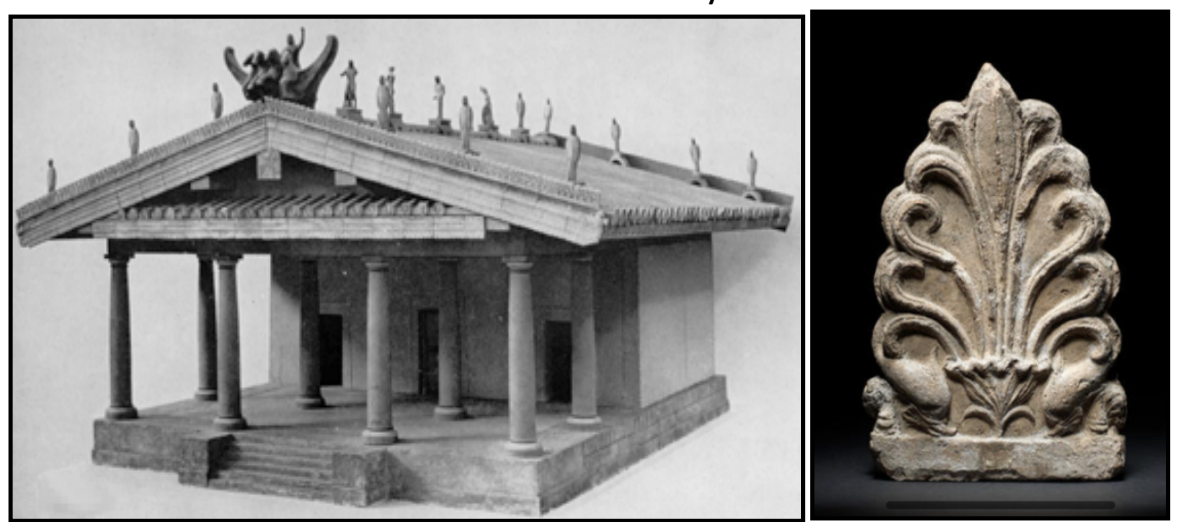

Figura 3: Reconstrucción del Templo de Portonaccio (Veio) en base a los restos encontrados. Fuente: es.khanacademy.org. Figura 4: Antefija del templo. Fuente: akrokerama.blogspot.com.es

En cuanto al carácter propio que alcanzó la cultura romana se debe en primer lugar a la victoria definitiva sobre los cartagineses (h. 202 A. C.), que les permitió dominar la zona occidental del Mar Mediterráneo y por consiguiente tener un mejor acceso a la zona del mar Egeo. Este hecho hizo posible que con los años tuviera lugar una gran aceptación de la cultura griega, mientras que durante la última centuria del primer milenio a. C. la cultura romana ya tenía un tipo de arquitectura propio, por lo que se podría considerar este periodo como el momento en el que la cultura romana hace su irrupción en el panorama del mundo conocido.

Así pues, de esta influencia griego-etrusca, los romanos al tener una mentalidad mucho más abierta y receptiva que sus predecesores, adquirirán y copiarán todos aquellos elementos tanto arquitectónicos como decorativos que consideraron útiles, para posteriormente mejorarlos. Por ejemplo, de la cultura etrusca se apropiarán la utilización del revestimiento de muros o el uso de en sus primeros edificios de cubiertas de teja y madera; en cambio de la cultura griega adoptaran el uso de las formas clásicas como formas de decoración u ornamentación.

Así pues, hay que hacer hincapié en el proceso de romanización que padecerán aquellos pueblos conquistados por los romanos, pues se verán obligados a aprender las leyes, costumbres, cultura, etc. romanas; y por ende sus "civitas et urbs" también asimilarán tanto el arte como la arquitectura predominante, cosa que se verá reflejada por todos los lugares del Imperio, ya que tomarán como modelo los edificios de la capital como por ejemplo el Teatro de Marcelo, las Termas de Trajano, el Anfiteatro Flavio, etc. 


\section{Cuerpo principal del estudio}

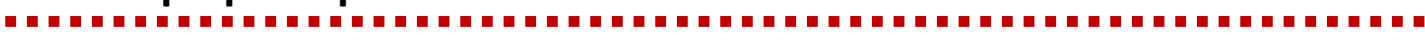

A continuación, vamos a abarcar lo que es el corpus principal de nuestro trabajo. En primer lugar, trataremos la temática forense, centrándonos en los foros de Tarraco y Emerita Augusta.

La Colonia de Tarraco, destacó por ser una de las capitales de provincia del Imperio lo que hacía que la ciudad estuviera dotada con una arquitectura de prestigio que ofrecía la doble condición de centro económico y político de primer nivel, puerto comercial, cabeza de conventus y capital comercial; así pues, el simple hecho de tener esta categoría, ya nos hace pensar en la magnitud de sus edificios y especialmente su foro, ya que es el espacio a tratar. El foro colonial, cómo podemos ver en el plano [Figura 5], se encontraba situado en la zona sur de la ciudad, cerca del mar, los restos allí encontrados se corresponden con una gran basílica forense de tres naves, con porticado perimetral de 14 x 4 columnas, gran tribunal central en el eje menor y pequeños locales anexos a ambos lados del mismo (Ruíz de Arbulo, 1990: 119-138), además, los trabajos de urbanización de la ciudad moderna en la parte baja de la ciudad permitieron la excavación de un gran conjunto porticado y un barrio anexo de casas y calles ((Ruiz de Arbulo, Mar, Domingo y Fiz, 2004: 123).

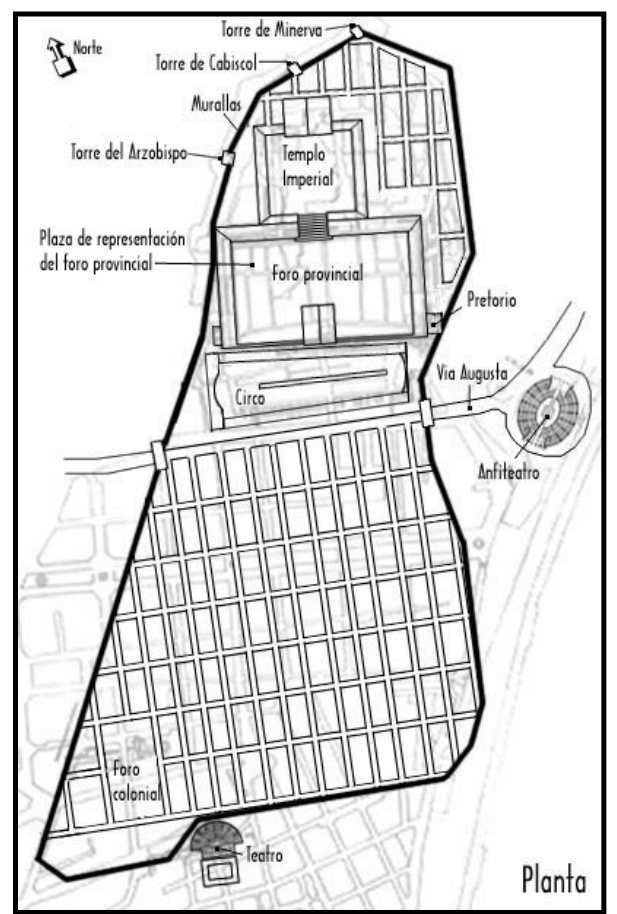

Figura 5: Plano de la Colonia Iulia Urbs Triumphalis Tarraco. Fuente: http://www.spanisharts.com.

Cuando tuvo lugar el cambio de era, es decir, el paso hacia la nomenclatura imperial, la ciudad tuvo que realizar una serie de reformas urbanas que hicieran palpable su condición de capital provincial; esto lo vemos reflejado en el momento en que los habitantes de Tarraco solicitarán al emperador Tiberio construir un Ara en memoria de Augusto 
y un templo en honor del propio emperador. Acerca de estos edificios todavía no se han hallado sus restos, pero si se han hallado monedas que nos hacen tener una idea sobre cómo era su parte frontal (Ruiz de Arbulo, 1990: 124-137).

Cambiando de lugar y de zona, como es bien conocido, Emerita Augusta fue fundada en el año 25 a.C. por orden del emperador Augusto para así poder licenciar a los soldados que habían combatido en las guerras cántabras. Al ser una ciudad de nueva planta en tiempos del primer César, es lógico que encontremos elementos de transición entre la época republicana y la época imperial. Así pues, el primer núcleo poblacional surgirá alrededor de lo que se denominará como forum coloniae o foro municipal [Figura 6]; el cual seguirá los cánones vitrubianos, siendo sus límites "el kardo maximus en el lado occidental, el decumanus maximus por el septentrional, el pórtico o forum adiectum por el oriental y por mediodía por algunos edificios, parte de los cuales,..., han sido descubiertos recientemente" (Álvarez Martínez y Nogales Basarate, 2004: 294).

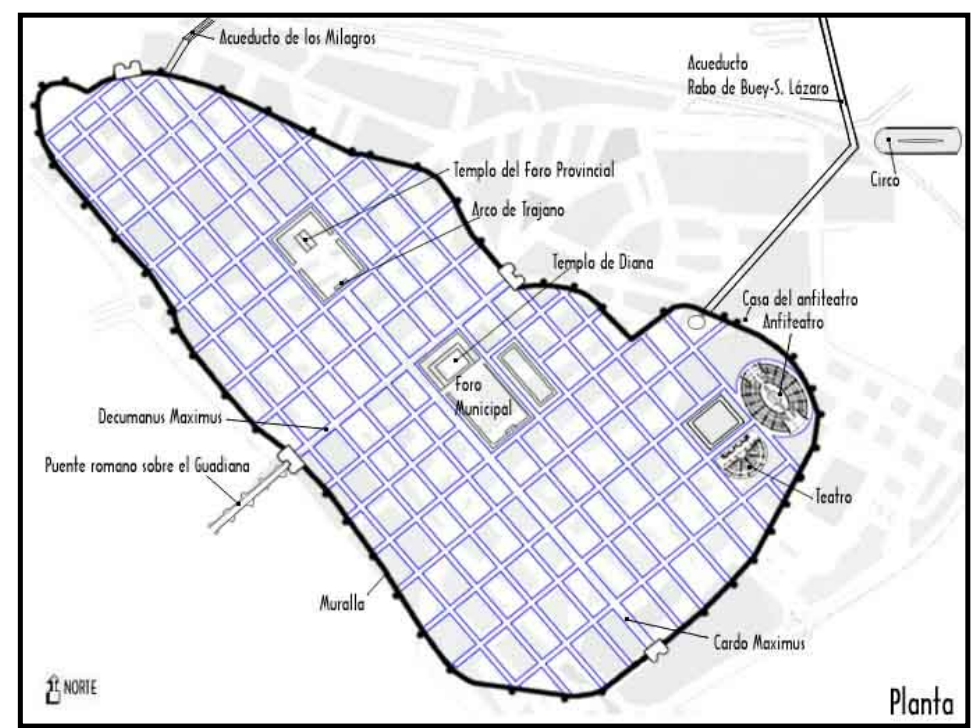

Figura 6: Planta de la Colonia Augusta Emerita. Fuente: http://ies.garciamorato.madrid.educa.madrid.org.

A día de hoy, no se han descubierto todos sus edificios, pero el más importante y mejor estudiado es el conocido como "Templo de Diana", el cual se sabe que pertenecía a este foro debido a la orientación de las escalinatas de acceso. El conjunto del templo de Diana también cuenta con un área sacra, probablemente ajardinada y con dos estanques a ambos lados del templo, respondiendo quizá a un mero hecho ornamental o decorativo; otro elemento arquitectónico que cabe destacar es el hallazgo de una tribuna, cuya longitud es idéntica a la anchura del pódium del templo, y su función sería la de un espacio donde tendrían lugar los actos ceremoniales en relación a la divinidad a la que estaba consagrada el templo, además de que también podría tener lugar algún acto de carácter civil o político, tal y como hacía Julio César en la tribuna del templo de Venus Genetrix. 
A nivel decorativo, se pueden visualizar elementos como los capiteles de orden corintio, realizados con una triple corona de acanto, destacando la decoración estucada, la cual está muy elaborada (Álvarez Martínez y Nogales Basarate, 2004: 303). Así pues, la fastuosidad de los restos hallados y conservados muestra la importancia de este sector de la antigua Emerita Augusta.

En el caso de los espacios lúdicos, se sigue el mismo patrón que en el caso de los conjuntos forenses, pues son obras sufragadas por ricas familias como ocurre con los Baebios en Sagunto.

En el caso de los edificios teatrales, estos empezarán a construirse de forma permanente en Roma a finales del periodo republicano, pues anteriormente estaban prohibidos por motivos de seguridad, de ahí que las representaciones tuvieran lugar en construcciones de madera decoradas con gran lujo. Posteriormente, en época imperial, este tipo de construcciones se extenderán por el resto de provincias con la intención de ofrecer al público divertimento y distracción; al ser edificios de índole imperial, en estos destacaría la decoración propagandística tanto del gobierno local como del imperial (Aranegui, 2004: 137-138).

Por lo que respecta a este tipo de edificios, me gustaría destacar el caso del teatro de Cartagena, pues sus cánones se corresponden con la arquitectura imperial propia del cambio de Era, convirtiéndose así en una pieza esencial en el proyecto de renovación urbana acaecido en época del emperador Augusto. Fue construido a imagen y semejanza de los grandes complejos teatrales de Roma, pues entre los materiales hallados encontramos capiteles corintios labrados en mármol de Cárrara (Itália) o columnas de travertino rosado (Ramallo Asensio et alii, 2010: 67-70).

Una de las principales funcionalidades de estos edificios de poder era la propaganda política, pues en lo que se correspondía con los accesos de entrada al teatro se encontraron dos dinteles conmemorativos [Figura 7] de caliza gris destinados a Cayo y Lucio César, nietos de Augusto. También podríamos hablar de función religiosa, puesto que aquí también se encontraron tres altares, que según los investigadores debieron estar situados en el frons pulpiti, donde están esculpidas tres aves que simbolizan la Tríada Capitolina: el águila, emblema de Júpiter, el pavo real, símbolo de Juno y la lechuza, símbolo de la diosa Minerva (Ramallo Asensio et alii, 2010: 79-87). 


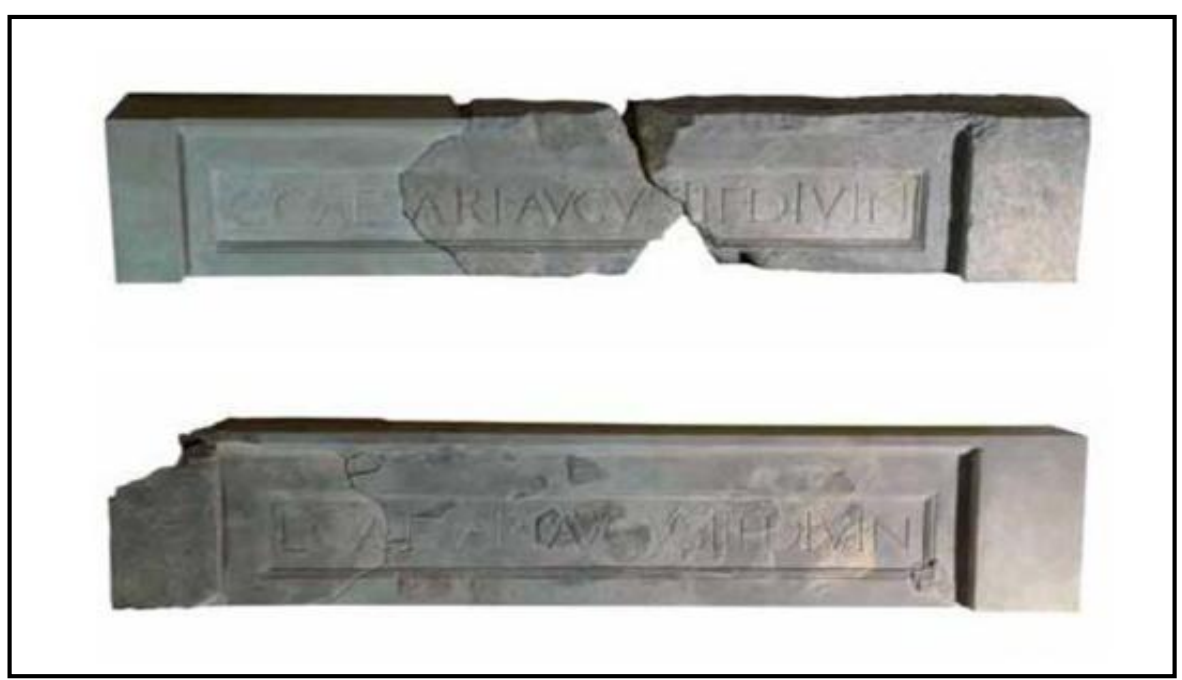

Figura 7: Dinteles conmemorativos del teatro de Cartagena (Ramallo Asensio et alii, 2010: 82)

En cuanto a los edificios que daban acogida a los ludii circenses, es decir, los anfiteatros, hay que destacar uno muy por encima de otros a nivel peninsular, debido a su excelente estado de conservación, gran capacidad y monumentalidad, concretamente, vamos a describir el anfiteatro de Italica [Figura 8], cuya capacidad se ha estimado en unas 25.000 personas. Este edificio de magnas dimensiones se encuentra situado en la zona norte de la ciudad, en la zona de extramuros siguiendo la línea de muralla; por lo que respecta a su fecha de construcción no se sabe a ciencia cierta, pero la mayoría de las teorías propuestas coinciden en que se levantó en tiempos del emperador Adriano, quien era nativo de esta ciudad. Una de las principales características que presenta este edificio, es que sigue el patrón del anfiteatro Flavio de Roma, pero con unas dimensiones más reducidas al tener un carácter más provincial, es decir, presentaría una estructura de opus caementicium revestido con losas de mármol, el graderío al igual que ocurre con los teatros estaría dividido en ima, media y summa cavea, siendo cada una de las zonas destinadas según la clase y el rango social, concretamente este anfiteatro conserva hasta la media cavea, además de contar con un podium que eleva la estructura para poder así obtener una mejor visión de la arena, en cuyo centro se halla la fossa bestiaria, la cual estaría recubierta por un entarimado de madera que se sustentaba por ocho pilares de ladrillo que todavía hoy perduran (Bellido Márquez, 2009: 34). El edificio en sí queda dividido en dos sectores que se corresponden con las puertas de acceso a la arena: la Porta Triumphalis, sita en la zona oriental, lugar de entrada de los gladiadores y salida del victorioso, y la Porta Libitienensis, localizada en la zona occidental, por la que salían aquellos gladiadores que eran derrotados en combate; acerca de esta última hay que destacar que se encuentra en un estado de conservación óptimo en comparación con la primera de ellas, llegando al punto en el que se puede apreciar el inicio de la superposición de los órdenes clásicos.

Debido a las características del relieve hispano, muchos de los teatros y anfiteatros de la Península se construían cerca de las laderas rocosas, por 
lo que el graderío era más fácil de construir y la acústica mejoraba notablemente. En el caso de este anfiteatro, se sigue esta disposición pues geográficamente está ubicado entre dos pequeñas colinas donde se sustentan las gradas. A pesar de estos factores positivos que presenta el anfiteatro de Italica, también hay que destacar otros negativos como por ejemplo el emplazamiento donde se encuentra situado, ya que la arena se ubicó sobre el curso de un arroyo para el cual se tuvo que construir unas canalizaciones a modo de cloaca por donde el agua fuera evacuada. Según Bellido Márquez, estas estuvieron en uso hasta hace, aproximadamente, unos treinta años, momento en que fueron inutilizadas tras largos años de desuso, un mantenimiento poco apropiado, el desuso y el expolio, sin olvidarnos de las inclemencias climáticas y las inundaciones (Bellido Márquez, 2009: 35).

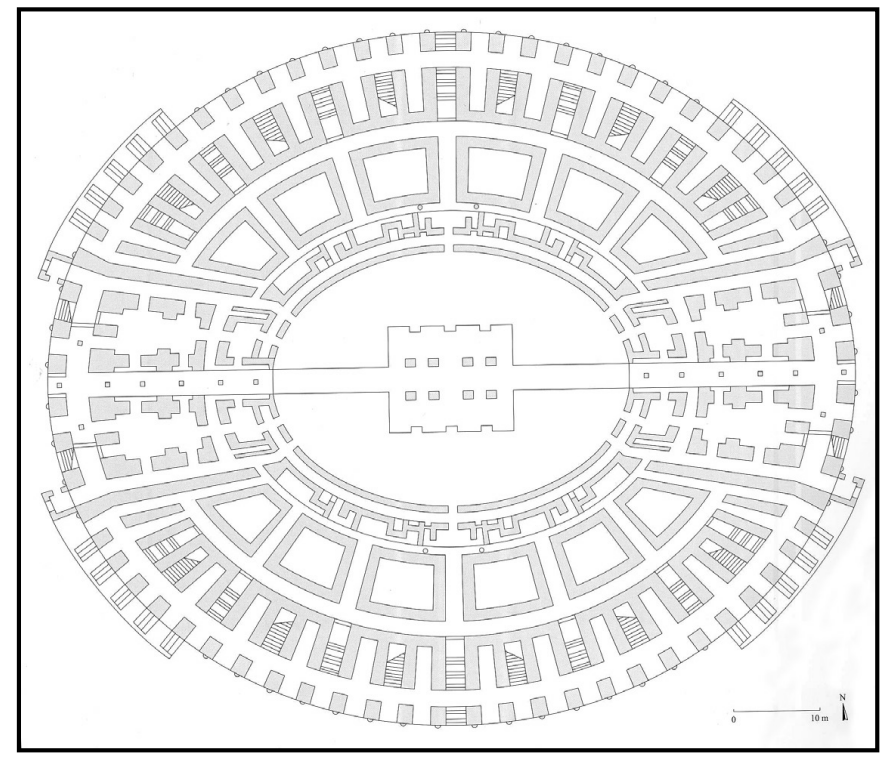

Figura 8: Planta del anfiteatro de Italica (Bellido Márquez, 2009: 36).

Para finalizar, abarcaremos otro tipo de restos arquitectónicos de prestigio para el mundo romano como fueron los edificios termales; a nivel Peninsular, numerosos han sido los restos termales hallados tanto de dominio público como privado.

Recientemente, en Baelo Claudia, se han hallado unas estructuras identificadas con unas termas suburbanas, habiéndose excavado una piscina del frigidarium y algunas estancias calefactadas, además de habitaciones con grandes dimensiones (Bernal et alii, 2013: 135). A nivel decorativo, cabe destacar la presencia de los restos de una estatua de mármol realizada a tamaño natural [Figura 9], además de placas de mármol de grandes dimensiones; estos materiales hallados junto a las dimensiones de las habitaciones excavadas, responden a la importancia que pudo tener su propietario ya que el mármol era un material de lujo que solo unos pocos se podían permitir (Bernal et alii, 2013: 137-138).

Los investigadores adscritos a este yacimiento descartan la idea de que se trate de una villa periurbana de grandes dimensiones, pues debido a la notable entidad de las estructuras balnearias que se han documentado 
hace pensar más en edificios de carácter público que en una residencia privada (Bernal et alii, 2013: 127). Esta teoría viene fundamentada por el hecho de que en toda la península no se tienen datos de que haya una gran villa o domus extra moenia de un gran asentamiento como Baelo Claudia, con unas termas de grandes dimensiones. Acerca de este edificio, todavía es pronto para sacar conclusiones puesto que todavía queda bastante por excavar.
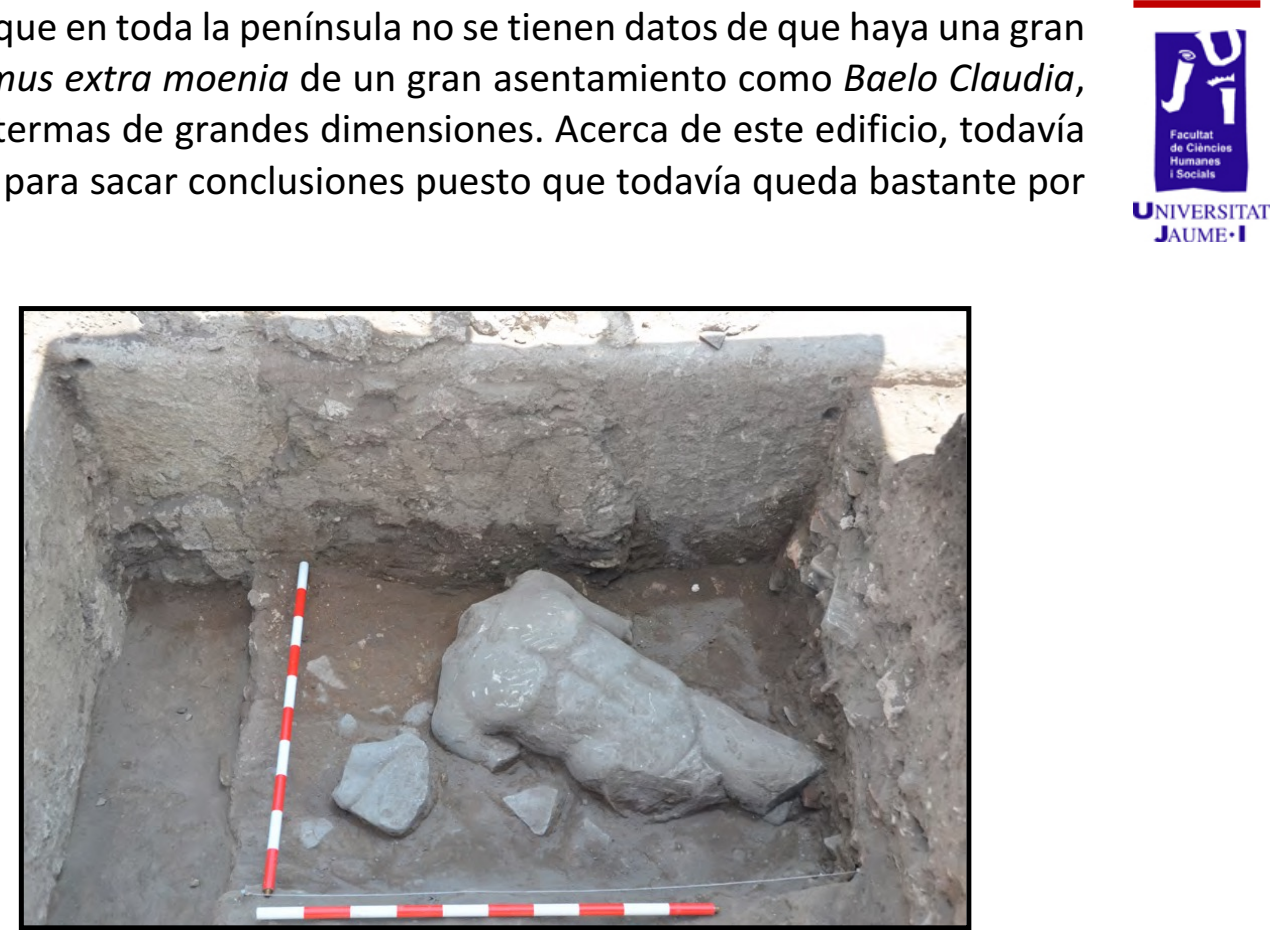

Figura 9: Restos escultóricos hallados en las termas extra moenia de Baelo Claudia (Bernal et alii, 2013: 137).

Otras termas peninsulares de renombre que merecen la pena ser tratadas son las de Clunia, conocidas como Los Arcos I y Los Arcos II [Figura 10 y 11$]$.

Los Arcos I, destaca por su monumentalidad y por ser un edificio axial simétrico, con la intención de destinar cada sección a un sexo. Su entrada tenía lugar a partir de una exedra semicircular porticada que daba acceso a unas palestras también porticadas desde donde se accedía al resto de estancias como el apodyterium, frigidarium, tepidarium o caldarium ((Núñez Hernández, 2008, 188-190).

De Los Arcos II, tan solo se dispone de un conocimiento parcial pues tan solo se ha excavado una parte de este yacimiento; concretamente se ha descubierto una gran palestra porticada tras la cual hay un gran apodyterium de planta octogonal, a través del cual se accede a un frigidarium con restos de pavimento de mosaico. El resto de salas se atribuyen a un tepidarium, caldarium y una sudatio ((Núñez Hernández, 2008, 173-175).

Por último, cabe destacar que ambos conjuntos termales estuvieron en uso durante la época imperial, esto, y conjuntamente a sus dimensiones espaciales, quiere decir que seguramente tuvieron una importante y suntuosa decoración, tal y como podemos observar en los pavimentos decorados de Los Arcos II, cosa que haría de ellas de uno de los edificios más representativos de la ciudad de de Clunia. 

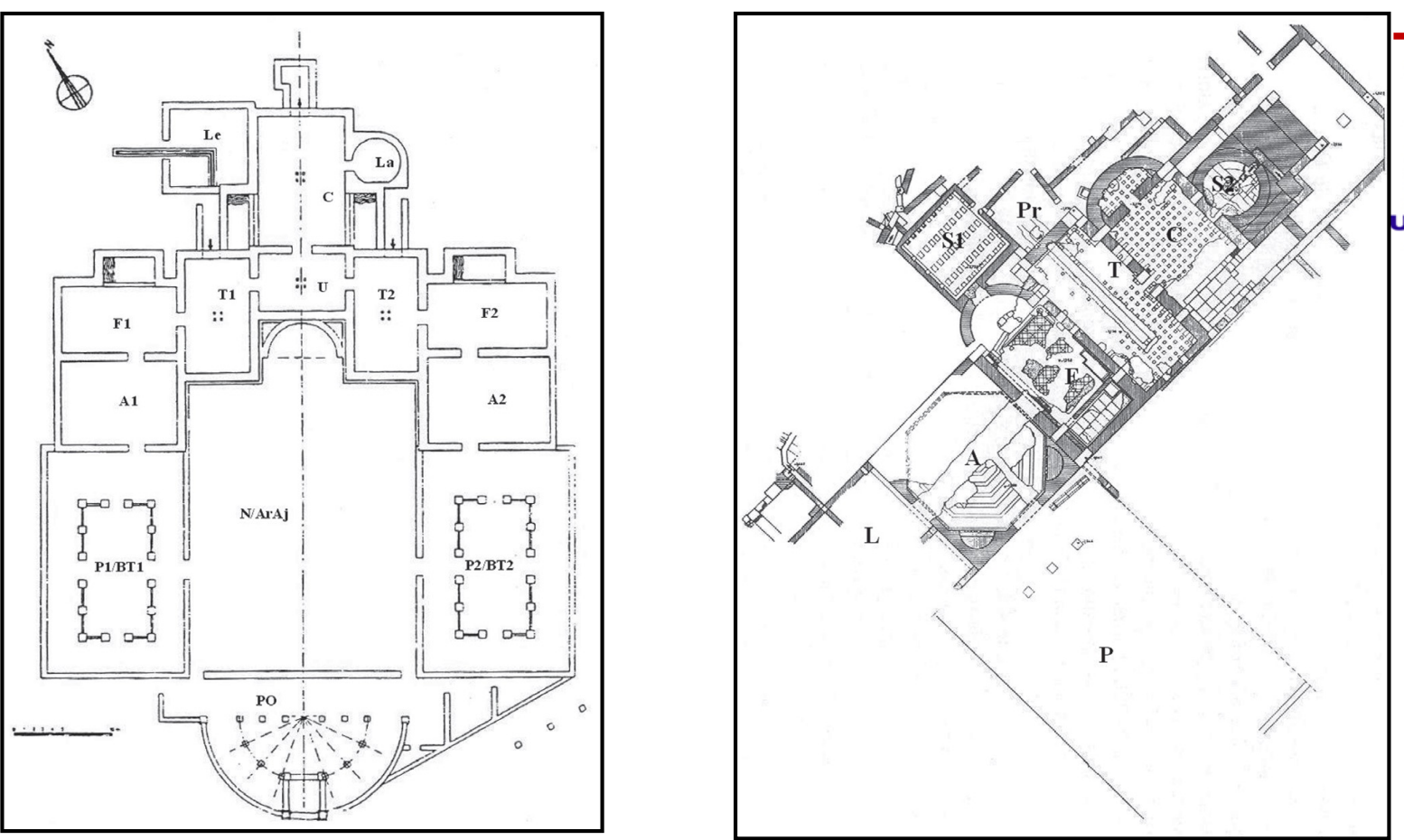

Figura 10: Plano de las termas de Los Arcos I (Palol, 1994: 81). Figura 11: Plano de las termas Los Arcos II (Palol et al., 1991: 370).

\section{Conclusiones}

Una de las principales conclusiones a las que he llegado al realizar este trabajo, es que el estudio de la arquitectura de poder reúne una problemática muy compleja, pues la bibliografía es muy abundante, y a su vez muy variada por lo que ha resultado complicado el buscar los artículos necesarios. Por otra parte, lo mismo ocurría al realizar el proceso de selección de aquellos elementos más adecuados, para poder indagar acerca de aquellos aspectos que nos interesaban para poder desarrollar este estudio, de ahí la complejidad de este trabajo.

Una de las cosas que más me ha llamado la atención es que a día de hoy, hay muchos estudios realizados acerca de la arquitectura de poder en época romana, aunque quizás no con esta nomenclatura. A pesar de ello, un hecho que merece la pena destacar es que cada autor sigue un modelo o patrón totalmente diferente a otro, por lo que podemos encontrar puntos como por ejemplo la ubicación del yacimiento o de un propio edificio al final o a mitad de nuestra lectura, cuando bajo mi punto de vista debería ir a principio, pues es necesario situarnos a nivel geográfico para tener en cuenta factores como el relieve u otros aspectos de similar índole.

Por este motivo, me gustaría proponer para finalizar un nuevo modelo de ficha de catalogación para las estructuras de poder romanas, siempre teniendo en cuenta sus posibles variables. 
Modelo de Registro de Arquitectura de Poder

\begin{tabular}{|c|c|c|}
\hline \multirow{3}{*}{ Arquitectura } & Sector & \multirow{3}{*}{ Tipo } \\
\hline & & \\
\hline & Fecha & \\
\hline
\end{tabular}

\section{Descripción morfológica}

Edificio lúdico

Edificio doméstico

Edificio público

Construcción conmemorativa

Otros

\section{Forma}

\section{Técnica}

\begin{tabular}{|l|l|}
\hline Materiales de construcción & Notas \\
\hline Opus testaceum & \\
\hline
\end{tabular}

Opus quadratum

Opus reticulatum

Opus caementicium

Opus signinum

Elementos decorativos

Opus tesellatum

Opus sectile

Opus vermiculatum

Opus marmoreum

Pintura mural

Otros 
- Apartado gráfico:

- Plano de localización:

- Cronología:

- Bibliografía:

\section{Bibliografía}

ADAM, J. P. (2002): La Construcción romana: materiales y técnicas, Editorial de los Oficios, León.

Álvarez Martínez, J. M. (1991): “El templo de Diana”, en Templos Romanos de Hispania. Cuadernos de Arquitectura Romana, Vol. I, pp. 83-93.

Álvarez Martínez, J. M. y Nogales Basarate, T. (2004): "Programas decorativos del Foro colonial de Augusta Emérita. El "Templo de Diana" - Templo de culto imperial", en Ramallo Asensio, S. F. (ed.): La decoración arquitectónica en las ciudades romanas de Occidente, Universidad de Murcia, pp. 293-319.

ARANegui Gascó, C. (2004): Sagunto: Oppidum, emporio y municipio romano, Edicions Bellaterra, Barcelona.

Bellido MÁrquez, T. (2009): “Panorama historiográfico del anfiteatro de Itálica", en Romula, no 8, Sevilla, pp. 33-64.

Bernal, D.; Arévalo, A.; Muñoz, A.; Expósito, J. A.; et alii (2013): “Las termas y el Suburbium marítimo de Baelo Claudia. Avance de un reciente descubrimiento", en Revista Onoba, no 01, pp. 115-152.

MAR, R. (1993): Els monuments provincials de Tarraco: noves aportacions al seu coneixement, Volumen 1, Universitat Rovira i Virgili, Tarragona.

MARÍn SÁnchez, R. (2000): La construcción griega y romana, Servicio de Publicaciones Universitat Politécnica de Valencia, Valencia.

Mateos CRUZ, P. (2009): Santuarios, oppida y ciudades: arquitectura sacra en el origen y desarrollo urbano del Mediterráneo occidental, Consejo Superior de Investigaciones Científicas. Instituto de Arqueología de Mérida, Volumen 45, Mérida.

Ministerio de Cultura (1987): Los Foros Romanos de las Provincias Occidentales, Dirección General de Bellas Artes y Archivos, Madrid. 
Oliver-BONJOCH I Oliver, J. (2004): Arquitectura y societat a la Roma Imperial, Barcelona, Edic. UPC.

NúÑez HernándeZ, S. I. (2008): “Conjuntos termales públicos en ciudades romanas de la cuenca del Duero", en Zephyrus, Universidad de Salamanca, LXII, Salamanca, pp. 163-193.

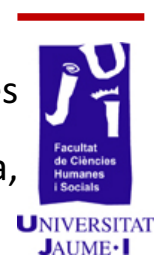

Palol Salellas, P. et. al. (1991): Clunia O. Studia Varia Cluniensia, Burgos.

Palol Salellas, P. (1994): Clunia. Historia de la ciudad y guía de las excavaciones, Burgos.

Ramallo Asensio, S. F. y Ruiz Valderas, E. (1998): El teatro romano de Cartagena, KR, Murcia.

Ramallo Asensio, S., Ruiz Valderas, E., Moneo Vallés, R. et alii (2010): Museo del Teatro Romano de Cartagena. Guía, Fundación Teatro Romano de Cartagena, Cartagena.

ROBERTSON, D. S. (1988): Arquitectura griega y romana, Cátedra, Madrid.

Ruiz de Arbulo, J.; MAR, R.; Domingo, J. y Fiz, I. (2004): “Etapas y elementos de la decoración arquitectónica en el desarrollo monumental de la ciudad de Tarraco (s. II a.C. - s. I d. C.)", en La decoración arquitectónica en las ciudades romanas de Occidente, de Ramallo Asensio, S. F. (ed.), Universidad de Murcia, pp. $115-151$.

Ruiz de Arbulo, J. (1990): “El foro de Tarraco”, Cysela, 8, Girona, pp. 119-138. WARD-PERKINS, J. B. (1989): Arquitectura Romana, Aguilar/Asuri, Madrid.

http://www.foroxerbar.com/viewtopic.php?t=9943 [02/01/2015].

https://lh5.googleusercontent.com/-

OiWzRRiVKqk/TYPwdS7sIKI/AAAAAAAABEc/I_4KfI8sFOc/Sestercio+Tito+Colis eo.jpg [02/01/2015].

http://www.wga.hu/index1.html [04/01/2015].

http://www.spanisharts.com/arquitectura/imagenes/roma/ciudad_tarraco_ plano.jpg [08/01/2015].

http://ies.garciamorato.madrid.educa.madrid.org//Dep_Griego/trabajos_ webquest/images/emerita_clip_image002.jpg [08/01/2015]. 
